Klimaschutzgesetz

\section{Nachsitzen für Freiheitsrechte}

Mit großer Aufmerksamkeit wurde in den vergangenen Tagen das Urteil des Bundesverfassungsgerichts aufgenommen, das den Kläger:innen gegen das 2019 verabschiedete Klimaschutzgesetz in Teilen Recht gab. Zwar wurde vom Gericht zugestanden, dass das Klimaschutzgesetz in weiten Teilen rechtskonform sei und keine grundrechtlichen Schutzrechte verletzt würden. Zugleich wurde jedoch festgestellt, dass das Gesetz die Freiheitsrechte jüngerer Menschen ungebührlich beschneiden würde. Der umfangreiche $\mathrm{CO}_{2}$-Verbrauch schon bis 2030, den das Gesetz erlaubt, verschärfe das Risiko schwerwiegender Freiheitseinbußen, weil damit die Zeitspanne für technische und soziale Entwicklungen knapper würden, mit deren Hilfe die Umstellung von der heute noch umfassend mit $\mathrm{CO}_{2}$-Emissionen verbundenen Lebensweise auf klimaneutrale Verhaltensweisen freiheitsschonend vollzogen werden könnte. Die $\mathrm{CO}_{2}$-Minderungspflichten, die zur Wahrung der Klimaziele von Paris (Begrenzung der globalen Durchschnittstemperatur auf deutlich unter $2^{\circ} \mathrm{C}$ und möglichst auf $1,5^{\circ} \mathrm{C}$ ) sowie zur Erreichung der Klimaneutralität in Deutschland bis 2050 erforderlich seien, würden die Möglichkeiten der jüngeren Generation ab 2031 zu sehr einschränken. Die Karlsruher Richter sehen hierin eine „umfassende Freiheitsgefährdung“ durch einseitige Verlagerung der Vermeidungslasten auf die Zukunft. Das Gericht zielt mit seinem Urteil zwar explizit auf die Versäumnisse in der Klimagesetzgebung nach 2030 ab, weil kein hinreichend konkreter „Fahrplan“ für den weiteren Weg zur Erreichung der Klimaziele festgelegt sei. Doch diese Feststellung beinhaltet zugleich Rückwirkungen auf den Klimaschutz bis 2030, wenn festgestellt wird, dass die gegenwärtigen Anstrengungen zu gering seien.

Hervorzuheben ist an dem Urteil vor allem, dass mehrfach und explizit auf den Ansatz des " $\mathrm{CO}_{2}$-Restbudgets“ zurückgegriffen wird, wie er vom Sachverständigenrat für Umweltfragen (SRU) entwickelt wurde. Dieses Restbudget zeigt an, wieviel Treibhausgase sich Deutschland unter den gesetzten Zielen noch leisten darf und wann bildlich gesprochen - die Badewanne überläuft. Der SRU hatte auf Grundlage eines gleichen Emissionsbudgets pro Weltbürger errechnet, dass für Deutschland ab 2020 ein „, $\mathrm{CO}_{2}$-Restbudget" von 6,7 Gigatonnen vorhanden sei. Die Politik hat diesen Ansatz bislang nicht aufgegriffen und sich stattdessen an prozentualen Minderungszielen

(c) Der/die Autor:in(nen) 2021. Open Access: Dieser Artikel wird unter der Creative Commons Namensnennung 4.0 International Lizenz veröffentlicht (creativecommons.org/licenses/by/4.0/deed.de).

Open Access wird durch die ZBW - Leibniz-Informationszentrum Wirtschaft gefördert. für Treibhausgasemissionen (bezogen auf 1990) orientiert. Damit aber werden die noch erlaubten Emissionsmengen nicht gut sichtbar. Nach SRU-Berechnungen müssten auf Basis des $\mathrm{CO}_{2}$-Restbudgets die Treibhausgase in Deutschland bis 2030 um $88 \%$ sinken, um das Pariser $1,5^{\circ} \mathrm{C}$-Ziel einzuhalten, beim $2^{\circ} \mathrm{C}$-Ziel wären es mindestens $68 \%$.

Der Ansatz des Gerichts, das $\mathrm{CO}_{2}$-Restbudget und damit quantitative Zielgrößen für die Ableitung von Maßnahmen zugrunde zu legen, steht in Einklang mit umweltökonomischen Empfehlungen, wonach Zielsetzungen im Umweltschutz tunlichst auf Gesamtemissionsmengen bezogen sein sollten. Mit einer solchen Festlegung tut sich die Politik bekanntermaßen nicht nur im Klimaschutz schwer; auch in anderen umweltpolitischen Feldern gibt es das bisher nicht. So gesehen ist das Urteil ein wichtiger Meilenstein nicht nur für den Klimaschutz.

Die Politik hat nun sehr kurzfristig reagiert, um nachzubessern. In Rede steht vor allem, anstatt der $55 \%$-Minderungen von Treibhausgasen bis 2030 nun $65 \%$ anzustreben. Bis 2040 sollen es dann $88 \%$ sein, und das Ziel der Klimaneutralität soll um fünf Jahre auf das Jahr 2045 vorgezogen werden. Soweit so gut. Es wird jedoch gewaltiger Anstrengungen bedürfen und die gesellschaftlichen Auseinandersetzungen nicht kleiner werden lassen, wenn es um die Umsetzung geht. Was das Herunterbrechen der neuen Ziele auf einzelne Sektoren anbetrifft, so steht zu befürchten, dass die Festlegung des Emissionsbudgets für Sektoren einem statischen Ansatz folgt, der Unterschiede in den Vermeidungspotenzialen oder den technologischen Innovationen in den einzelnen Sektoren und - damit verbunden - Unterschiede in den Grenzvermeidungskosten außer Acht lässt. Es ist wenig sinnvoll und auch nur schwer umsetzbar, wenn beispielsweise der Gebäudesektor bis 2049 eine bestimmte Menge an Treibhausgasen einsparen soll, insbesondere wenn dies in anderen Sektoren zu weitaus geringeren Kosten möglich wäre. Hinzu kommt, dass die Rolle der negativen Emissionstechnologien, um Treibhausgase der Atmosphäre zu entziehen, bisher völlig unklar ist. Aus ökonomischer Sicht ist die Politik daher gut beraten, vor allem auf den $\mathrm{CO}_{2}$-Preis zu setzen. Es gilt, den Emissionshandel auszubauen, $\mathrm{CO}_{2}$-Preise für die Sektoren außerhalb des Emissionshandels festzulegen und Kompensation zwischen verschiedenen Sektoren zuzulassen. Gerade der Ruf nach flexibleren Instrumenten ist vor dem Hintergrund der neuen ambitionierten Zielsetzungen dringender denn je.

Bernd Hansjürgens Helmholtz-Zentrum für Umweltforschung - UFZ bernd.hansjuergens@ufz.de 